\title{
Density functional study of interactions between fluorinated cyclohexanes and arenes $\dagger$
}

\author{
Rodrigo A. Cormanich, ${ }^{\text {a,b }}$ Alastair Durie, ${ }^{\text {a }}$ Ragnar Bjornsson, ${ }^{\text {c }}$ Roberto Rittner, \\ David O'Hagan $^{\mathrm{a}^{*}}$ and Michael Bühl ${ }^{\mathrm{a}^{*}}$
}

${ }^{a}$ EastChem School of Chemistry and Centre of Magnetic Resonance, University of St Andrews, North Haugh, St Andrews, Fife, KY16 9ST, UK Fax: 01334 463800; Tel: 01334467171 ;

${ }^{\mathrm{b}}$ Chemistry Institute, University of Campinas, Campinas, SP, 13083-970, Brazil, Tel (19) 3521 3150; Fax. (19) 35213023

${ }^{\mathrm{c}}$ MPI for Chemical Energy Conversion, Stiftstraße 34 - 36, D-45470, Mülheim an der Ruhr, Germany.

E-mail: $\underline{\text { mb105@st-andrews.ac.uk and do1@st-andrews.ac.uk }}$

\begin{abstract}
${ }^{1} \mathrm{H}$ NMR chemical shifts of all-syn-1,2,4,5-tetrafluorocyclohexane (1), all-syn-1,2,3,4tetrafluorocyclohexane (2) and their complexes with benzene are calculated at the BHandH/6-311+G(2d,p) level. The observed shielding of certain resonances on going from $\mathrm{CD}_{2} \mathrm{Cl}_{2}$ to toluene- $\mathrm{d}_{8}$ solution is qualitatively reproduced in these model calculations, in particular when standard B3LYP/def2-TZVP optimised geometries are employed. The results are interpreted with the quantum theory of atoms in molecules (QTAIM) and non-covalent interactions (NCI) methods, and indicate that aromatic molecules bind strongly $\left(1.6 \mathrm{kcal} \mathrm{mol}^{-1}\right)$ to the "positive face" of these molecules. The level of theory is validated for the new compound all-syn-3-phenyl-1,2,4,5tetrafluorocyclohexane (3), where a recently developed QM/MM protocol for optimisation of molecular crystals afforded excellent agreement between the B3LYP/def2-TZVP structure and that observed in the solid.
\end{abstract}




\section{Introduction}

Fluorine compounds have been widely used in many fields, with emphasis in medicinal and material sciences. The inclusion of fluorine atoms and/or fluorinated groups provide new and unusual molecular properties, which indeed can be used to obtain more active and selective drugs and materials with improved efficiency. ${ }^{1,2}$ In this sense, Durie et al. reported the synthesis of the all-syn-1,2,4,5-tetrafluorocyclohexane (1) and the all-syn-1,2,3,4-tetrafluorocyclohexane (2, Scheme 1), ${ }^{3,4}$ which were shown to have high polarity and low viscosity, and, hence, are potentially new candidates to be used as liquid crystal materials. Some ${ }^{1} \mathrm{H}$ NMR resonances of $\mathbf{1}$ and $\mathbf{2}$ showed unusually strong upfield shifts on going from $\mathrm{CD}_{2} \mathrm{Cl}_{2}$ to toluene solution, which were not fully understood at the time. ${ }^{3}$ We now report theoretical calculations for $\mathbf{1}$ and $\mathbf{2}$ using simple solvation models in order to highlight the specific interactions with aromatic solvent molecules which give rise to these unusual upfield shifts.

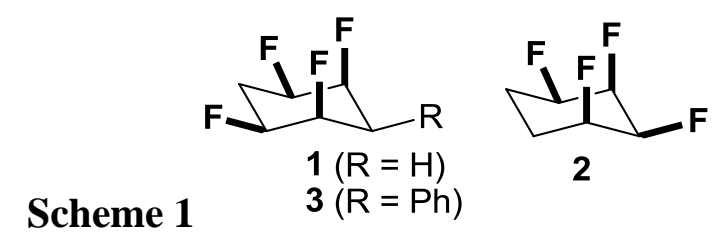

A model for the interaction between the fluorinated cyclohexane moieties and aromatic groups was provided by the recent synthesis and characterisation of all-syn-3phenyl-1,2,4,5-tetrafluorocyclohexane (3). ${ }^{5}$ Using a suitable computational protocol, we now report on the effect of intermolecular packing on the ${ }^{1} \mathrm{H}$ chemical shifts in $\mathbf{3}$, which is of interest in relation with the abovementioned solvent shifts in $\mathbf{1}$ (and 2).

\section{Results and Discussion}

The experimental ${ }^{1} \mathrm{H}$ NMR chemical shifts of $\mathbf{1}$ and $\mathbf{2}$ in $\mathrm{CD}_{2} \mathrm{Cl}_{2}$ and toluene- $\mathrm{d}_{8}$ of allsyn-1,2,4,5-tetrafluorocyclohexane (1) and all-syn-1,2,3,4-tetrafluorocyclohexane (2) are included in Table 1. The shielding of the ${ }^{1} \mathrm{H}$ resonances on going from dichloromethane to toluene is a manifestation of the well-known aromatic solvent induced shift (ASIS), ${ }^{6}$ albeit of exceptional magnitude, as individual chemical-shift changes reach up to $\Delta \delta=1.7 \mathrm{ppm}$. In order to rationalize these findings, we performed 
density functional theory (DFT) calculations for $\mathbf{1}$ and $\mathbf{2}$ (Figure 1), as well as their complexes with a single benzene molecule (as a model for toluene) ${ }^{7}$ attached at the "positive face", i.e., on the opposite molecular side of the axial fluorine atoms (Fig. 2).

Table 1: Experimental and theoretical $[\mathrm{BHandH} / 6-311+\mathrm{G}(2 \mathrm{~d}, \mathrm{p})]^{a}{ }^{1} \mathrm{H}$ chemical shifts $(\delta$ in ppm) for compounds $\mathbf{1}$ and $\mathbf{2}$ (atom numbering see Figure 1).

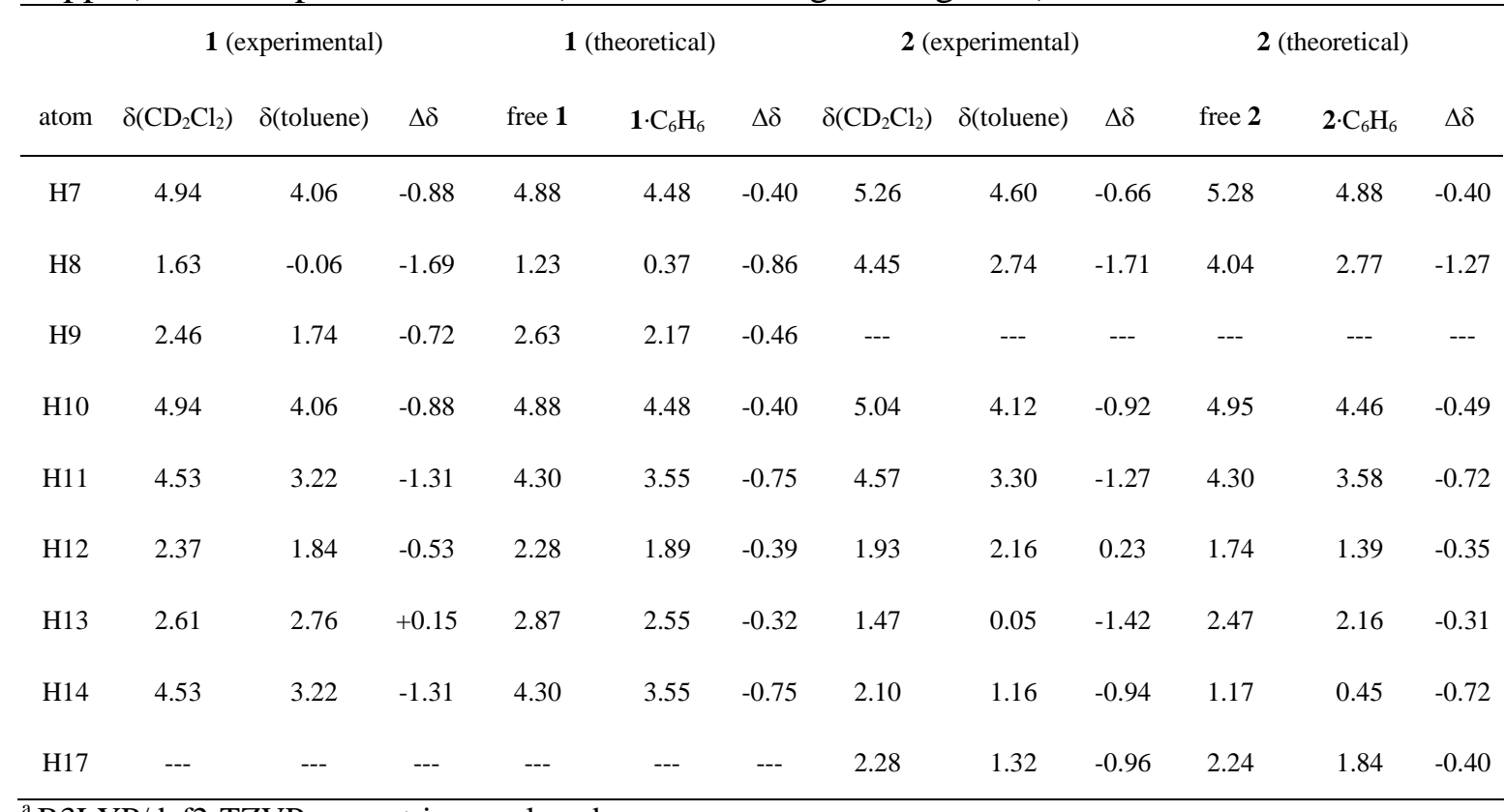

${ }^{\mathrm{a}}$ B3LYP/def2-TZVP geometries employed.
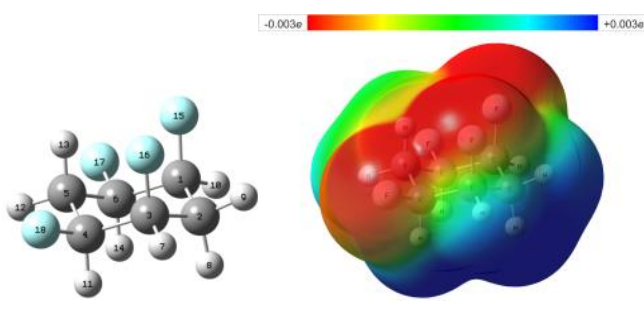

$1(\mu=4.49 \mathrm{D})$
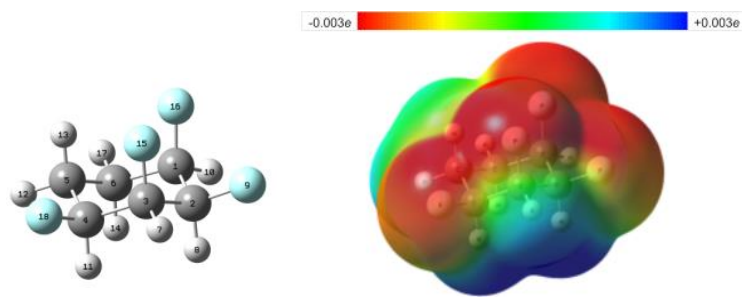

$2(\mu=4.71 \mathrm{D})$

Figure 1: Optimised structures, electrostatic potentials (ESPs) and dipole moments of 1 and 2 [B3LYP/def2-TZVP]. ESPs are plotted on a color scale from - 0.03 a.u. (red) to +0.03 a.u. (blue) and mapped onto an isodensity surface $\left(\rho=4 \cdot 10^{-4}\right.$ a.u. $)$

Both 1 and 2 have large dipole moments and highly polarised charge distributions that are very negative at the molecular face containing the fluorine atoms ("negative face"), and positive at the opposite face ("positive face", see ESPs in Figure 1). The electronrich aromatic solvent molecules bind preferentially to the positive faces (with a binding 
energy of ca. $1.6 \mathrm{kcal} \mathrm{mol}^{-1}$ for both 1 and 2 at the B3LYP/def2-TZVP ${ }^{8}$ level). This result is confirmed by the molecular graphs obtained from the quantum theory of atoms in molecules (QTAIM) ${ }^{9}$ and an analysis based on the recently developed non-covalent interactions (NCI) method ${ }^{10}$ (Figure 2). The latter has been developed from the former in order to overcome ambiguities in the classification of weak interatomic interactions as attractive or repulsive, which is achieved by performing the topological analysis of the reduced density gradient (RDG) instead of the electron density

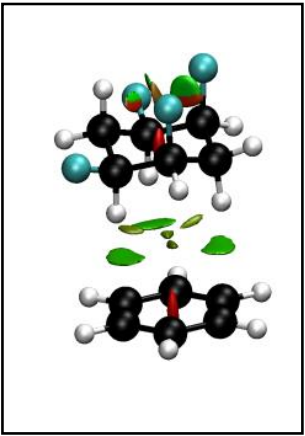

1 (QTAIM) ( $\rho)$ itself.

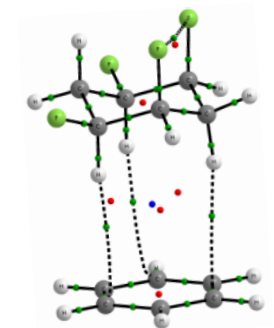

1 (NCI)

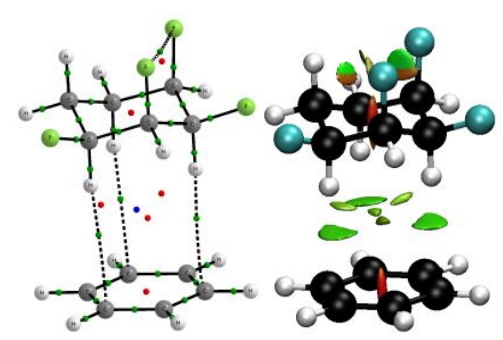

2 (QTAIM) 2 (NCI)

Figure 2: QTAIM and NCI molecular graphs obtained from the B3LYP/def2-TZVP electron density; green, red and blue points in the QTAIM graphs represent bond critical points (BCPs), ring critical points (RCPs) and cage critical points (CCPs), respectively. NCI surfaces are green for attractive van-der-Waals interactions and red for internal ring electron density depletion/repulsion (analogous to ring-critical points in QTAIM). NCI plots were obtained with $\mathrm{RDG}=0.6$ and blue-green-red scale ranging from -0.04 to $0.04 \mathrm{au}$.

a) compound 1

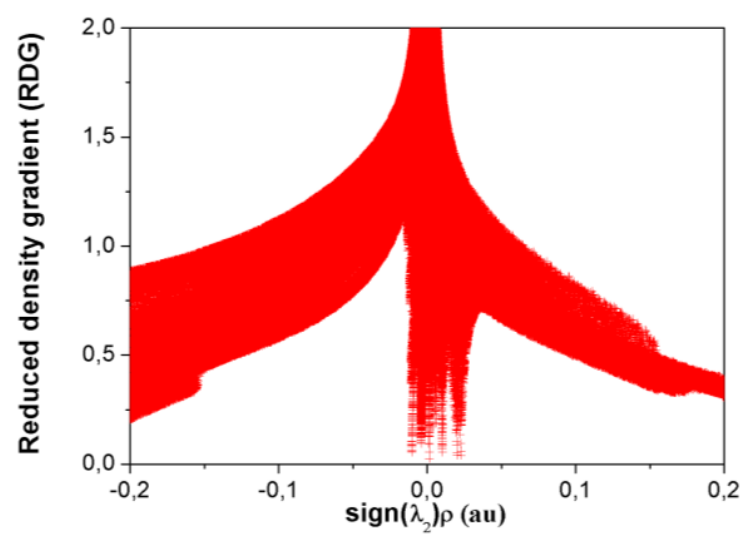

b) compound 2

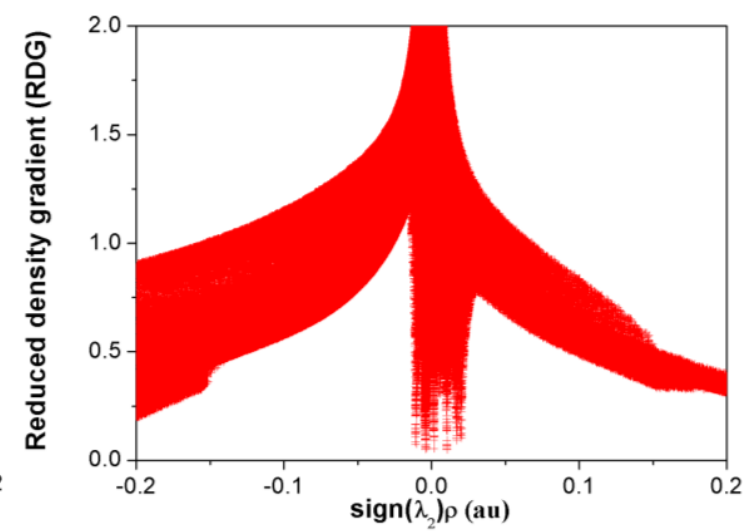

Figure 3: Plot of the reduced density grandient (RDG) versus $\operatorname{sign}\left(\lambda_{2}\right) \rho$ for (a) compound 1 and (b) compound 2 [sign $\left(\lambda_{2}\right)$ meaning the sign (positive or negative) of second eigenvalue $\lambda_{2}$ of the Laplacian of the electron density $\rho$ ]. Downward peaks at negative $\operatorname{sign}\left(\lambda_{2}\right) \rho$ values indicate attractive non-covalent interactions in the regions 
between the axial $\mathrm{F}$ atoms, downward peaks at positive $\operatorname{sign}\left(\lambda_{2}\right) \rho$ values indicate ring/cage critical point regions.

Both methods indicate not only formation of $\pi \cdots \mathrm{H}-\mathrm{C}$ intermolecular interactions between the cyclohexane and benzene moieties (for details of their QTAIM characterisation see Table S1 in the ESI), but also intramolecular F $\cdots$ F interactions within 1 and $\mathbf{2}$ (also for the free molecules, not shown). Such interactions have attracted much interest and controversy in the literature. ${ }^{11,12}$ According to our NCI analysis (see Figure 3) these interactions are classified as attractive.
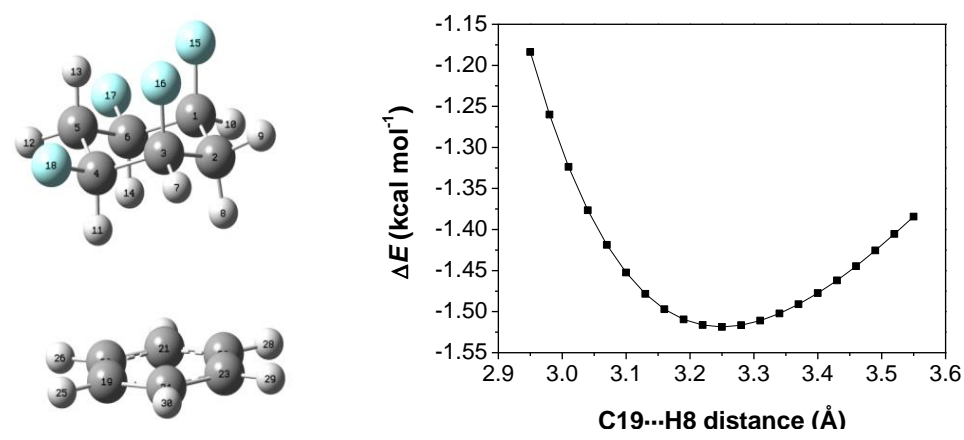

\section{$\mathbf{1} \cdot \mathrm{C}_{6} \mathrm{H}_{6}$}
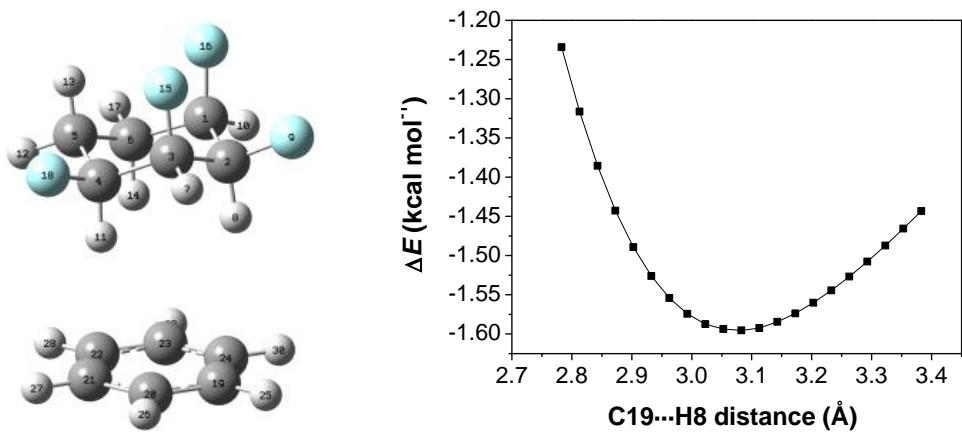

$\mathbf{2} \cdot \mathrm{C}_{6} \mathrm{H}_{6}$

Figure 4: Optimised complexes of $\mathbf{1}$ and $\mathbf{2}$ with benzene and interaction energies at the B3LYP/def2-TZVP level.

Solute-solvent interaction potentials were obtained through relaxed scans of salient distances between aliphatic $\mathrm{H}$ and aromatic $\mathrm{C}$ atoms in steps of $0.03 \AA$ at the B3LYP and B97-D levels (B3LYP results shown in Figure 4). The B97-D level was chosen in addition to the popular B3LYP functional because it was suspected that dispersion interactions would be very important. Indeed, on going from B3LYP to B97-D the minimum deepens considerably (from ca. $-1.6 \mathrm{kcal} / \mathrm{mol}$, see Figure 4 , to ca. $-6 \mathrm{kcal} / \mathrm{mol}$, 
see Figure S1) and is shifted to shorter distances (from ca. 3.1-3.2 $\AA$ to ca. 2.6-2.7 $\AA$ ). Essentially the same results are obtained when the empirical dispersion corrections ${ }^{13,14}$ are added as single points to the structures of the B3LYP scan (see B3LYP-D and B3LYP-D3 data in Figure S1).

The ${ }^{1} \mathrm{H}$ chemical shifts were calculated both for free molecules $\mathbf{1}$ and $\mathbf{2}$ and for their complexes with benzene, using B3LYP and B97-D geometries. It was expected that the difference between the isolated molecules and the benzene complexes could be related to the observed solvent shifts on going from $\mathrm{CH}_{2} \mathrm{Cl}_{2}$ to toluene. In fact the results for both sets of structures (B3LYP and B97-D) follow the observed trends reasonably well in a qualitative sense, in particular for compound $\mathbf{1}$, where the relative order shifts of the individual signals is well reproduced (see filled circles in the plot in Figure 5). Interestingly the shifts calculated for the B3LYP structures afforded a slightly better qualitative agreement with experiment (compare the $\Delta \delta$ values in Table 1) than those calculated for the tighter B97-D minima (Tables S2-S5). For the latter, individual deviations between modeled and observed solvent shifts can reach up to ca. 2 ppm (e.g. for H8 in compound 2, Table S5).

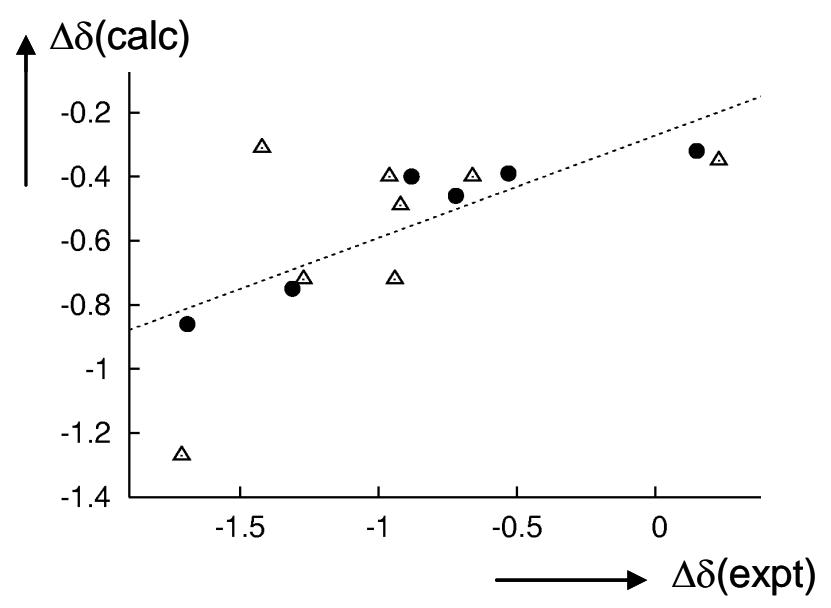

Figure 5: Plot of calculated shifts in ${ }^{1} \mathrm{H}$ resonances upon complex formation with benzene vs. observed shift differences on going from $\mathrm{CH}_{2} \mathrm{Cl}_{2}$ to toluene solutions (in ppm); filled circles: compound $\mathbf{1}$, open triangles: compound $\mathbf{2}$.

As a consequence of such complexation of $\mathbf{1}$ and $\mathbf{2}$ with benzene, the benzene ring current ${ }^{15}$ effectively shields those hydrogen atoms in its "shielding cone" perpendicular 
to the molecular plane. It is thus the hydrogen atoms lying in the positive molecular ESP area, i.e., atoms H8, H11 and H14, which experience the highest shielding effect, since these interact strongly with the solvent and, hence, are closer to the shielding cone of the aromatic rings. As mentioned above, when dispersion corrections are included in calculating the alkane-arene interaction energies (either via B3LYP-D3 single points or at the B97-D level), the minima in Figure 4 deepen and shift to lower distances (Figure S1). The chemical shifts are sensitive to this distance and deteriorate noticeably for such dispersion-corrected minima. Apparently B3LYP (which describes the leading electrostatic contributions correctly) affords reasonable models for averaged (rather than equilibrium) structures in these cases.

For a more quantitative description, one would have to include more solvent molecules and model their time-averaged effect on the solute shifts, e.g. by way of molecular dynamics simulations. That our simple theoretical model can already capture a large part of the observed effect can be taken as evidence for a predominance of solute-solvent complexes like those in Figure 2 in the dynamic ensemble in solution. Note that the observed pattern in the solvent shifts is due to ring-current effects in such solute-solvent complexes, rather than to bulk solvation effects. This is demonstrated by repeating the NMR calculations for the monomer immersed in a polarisable continuum (PCM) modeling benzene. Relative to the gas phase results, the obtained NMR chemical shifts are deshielded rather uniformly without distinguishing protons from either face (by ca. $0.3 \mathrm{ppm}$ and $0.2 \mathrm{ppm}$ for $\mathbf{1}$ and $\mathbf{2}$, respectively, see Table S6).

a)

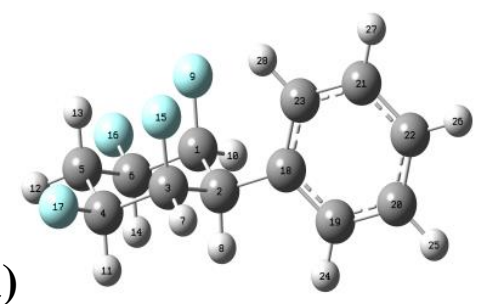

b)

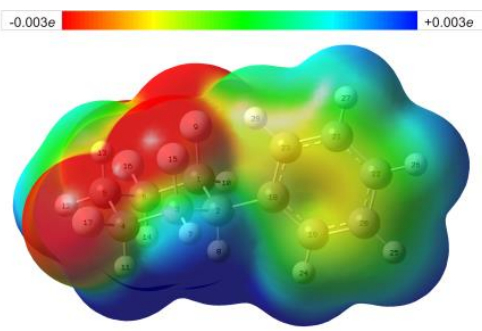

c)

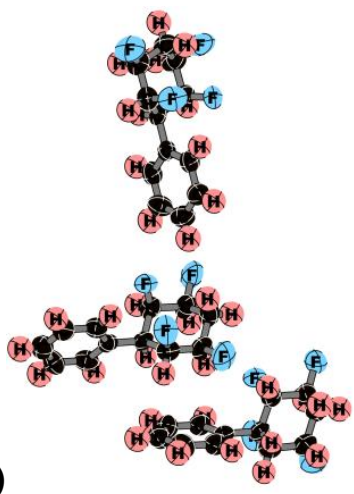

Figure 6: (a) B3LYP/def2-TZVP optimised structure of compound 3, (b) its calculated ESP, showing the "positive face" (lower molecular part) and "negative face" (upper molecular part, see caption of Figure 1 for details) and (c) experimental X-ray structure showing the interaction of a phenyl group located in the lower molecule with the "positive face" of the cyclohexane ring of the central molecule in an almost parallel 
arrangement; note the interaction between the "negative face" of the central cyclohexane ring with another phenyl group from the upper molecule in a perpendicular arrangement.

Recently the synthesis and structure of all-syn-3-phenyl-1,2,4,5tetrafluorocyclohexane (3) was reported..$^{5}$ This compound is an analogue of $\mathbf{1}$, which was also proposed as a new structural motif for liquid crystals. This compound turns out to be a good model to analyze the complexation of $\mathbf{1}$ with aromatic groups, since it was possible to obtain the X-ray structure for $\mathbf{3}$ and it was observed that the phenyl groups interact with the "positive face" of cyclohexane rings in neighboring molecules in almost the same geometry we have obtained for 1 (Figure 6c).

Comparing the observed intermolecular cyclohexane-arene distance in $3(\mathrm{C} \cdots \mathrm{H} \approx$ $3.46 \AA$ ) with that in $\mathbf{1} \cdot \mathrm{C}_{6} \mathrm{H}_{6}$, the B3LYP-optimised values for the latter are closer (ca. $3.25 \AA$, left part of Figure 2) than the B97-D data (ca. 2.71 $\AA$, left part in Figure S1). To probe whether the apparent good performance of B3LYP holds for the intermolecular interactions in $\mathbf{3}$ as well, we optimised the full molecular crystal employing the recently developed QM/MM protocol by Björnsson and Bühl (see Computational Details) ${ }^{16}$ together with a variety of standard functionals with and without explicit dispersion corrections. Key interatomic distances obtained by each method and from experiment are shown in Tables S7-S8 in the ESI. Compared to pristine $\mathbf{1} \cdot \mathrm{C}_{6} \mathrm{H}_{6}$ in the gas phase, inclusion of dispersion corrections has a much smaller effect on the intermolecular distances in solid 3. For example, on going from B3LYP/def2-TZVP to B3LYPD3/def2-TZVP, most intermolecular distances between the cyclohexane and the neighboring phenyl moieties decrease slightly, by less than $0.03 \AA$ (see C…C distances in Table S8), i.e. by much less than for the pristine benzene complexes $\mathbf{1} \cdot \mathrm{C}_{6} \mathrm{H}_{6}$ and 2. $\mathrm{C}_{6} \mathrm{H}_{6}$ discussed above. Overall, the B3LYP/def2-TZVP level performs best in reproducing the structure in the solid, as judged by the root-mean-square deviation (RMSD) between the optimised and observed coordinates of all $\mathrm{C}$ and $\mathrm{F}$ atoms in the trimeric QM region (ca. $0.04 \AA$, Figure 7a). All other level/basis-set combinations (including dispersion-corrected functionals) afford slightly higher RMSDs, up to ca. $0.08 \AA$ (Table S8). 
The effect of the crystal environment on the relative orientation of the molecular interactions can be probed by relaxing a section, e.g. the trimer shown in Figure $7 \mathrm{a}$, in the gas phase. As can be seen in Figure 7b, the B3LYP/def2-TZVP minimum of this trimer in the gas phase is noticeably distorted from that in the crystal.
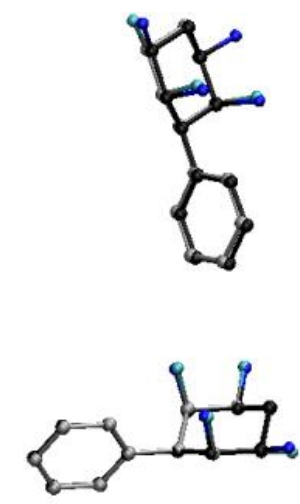

a)

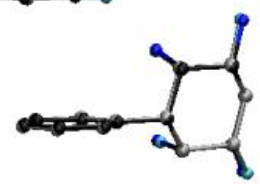

RMSD $=\mathbf{0 . 0 3 6}$
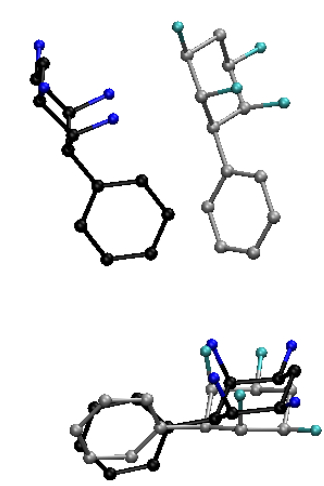

b)

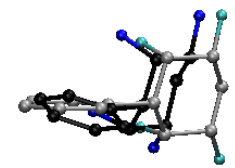

RMSD $=1.207$

Figure 7: Superposition of a section of the experimental crystal structure of compound 3 (grey/light blue) with (a) the same section from the QM/MM optimised crystal (black/dark blue) and (b) gas phase calculated geometries (B3LYP/def2-TZVP; black/dark blue), including root-mean-square deviation between both (RMSD, in $\AA$ ).

Table 2: Theoretical [BHandH/6-311+G(2d,p)] ${ }^{1} \mathrm{H}$ isotropic magnetic shieldings $(\sigma)$ and chemical shifts $(\delta)$ in ppm for free monomeric 3 and for the central molecule in the QM trimer within the QM/MM optimised crystal of $\mathbf{3}$ (B3LYP/def2-TZVP), together with experimental data in solution (atom numbering as in Figure 6).

\begin{tabular}{|c|c|c|c|c|c|c|}
\hline Hydrogen & $\sigma($ monomer $)$ & $\delta(\text { monomer })^{[\mathrm{a}]}$ & $\sigma($ complex $)$ & $\delta(\text { complex })^{[a]}$ & $\Delta \delta$ & $\begin{array}{c}\delta\left(\mathrm{CDCl}_{3}\right) \\
\text { Experimental }\end{array}$ \\
\hline $\mathrm{H} 7, \mathrm{H} 10$ & 26.04 & 5.58 & 26.44 & 5.18 & -0.40 & 5.05 \\
\hline $\mathrm{H} 8$ & 29.00 & 2.62 & 29.16 & 2.46 & -0.16 & 2.63 \\
\hline H11,H14 & 26.79 & 4.83 & 28.65 & 2.97 & -1.86 & 4.62 \\
\hline $\mathrm{H} 12$ & 28.80 & 2.82 & 29.31 & 2.31 & -0.51 & 2.81 \\
\hline H13 & 28.13 & 3.49 & 29.44 & 2.18 & -1.31 & 2.51 \\
\hline
\end{tabular}

${ }^{[a]} \delta=\sigma_{\text {TMS }}(31.62 \mathrm{ppm})-\sigma_{\mathrm{H}}$

${ }^{1} \mathrm{H}$ NMR chemical shifts for 3 were obtained at the BHandH/6-311+G(2d,p) level for a single isolated molecule and for the central molecule embedded in the trimer 
(B3LYP/def2-TZVP QM/MM geometries, Table 2). The data for the trimer are in good agreement with those of compound $\mathbf{1}$ complexed with benzene (compare values in Tables 1 and 2). Also, the computed trends upon complex formation for compound $\mathbf{3}$ are similar for that obtained for compound 1. The ${ }^{1} \mathrm{H}$ NMR chemical shift calculations for monomeric 3 also agree with the experimental values obtained in $\mathrm{CDCl}_{3}$ (Table 2). These findings confirm that, as expected, the intermolecular contacts seen in the crystal are all lost upon dissolution in $\mathrm{CDCl}_{3}$ (i.e. no smaller aggregates are preserved). The $\Delta \delta$ values between monomer and complex in Table 2 can serve as predictions for the expected solution-to-solid shifts once solid-state NMR studies of compound $\mathbf{3}$ become available.

\section{Conclusion}

Overall, simple theoretical models can rationalise the experimentally observed chemical shift values in toluene solution for the compounds $\mathbf{1}$ and $\mathbf{2}$. The leading contribution is indicated to arise from close contact between an aromatic ring from the solvent and the "positive face" of the cyclohexane ring opposite to the fluorine substituents. The qualitative trends are reproduced for simple complexes between $\mathbf{1}$ or $\mathbf{2}$ and a single benzene molecule at the B3LYP level, i.e. even without inclusion of dispersion corrections. This level has also been validated using a recently developed QM/MM protocol for the optimisation of molecular crystals, where excellent agreement was found between the B3LYP-computed and the observed crystal structure for the new compound $\mathbf{3}$, which is governed by such intermolecular cyclohexane $\cdots$ arene interactions. The attractive nature of these interactions is also apparent in a recently proposed analysis based on the Quantum Theory of Atoms in Molecules approach, namely the Non-Covalent Interactions (NCI) method.

\section{Computational Details}

Geometries were fully optimized at the B3LYP/def2-TZVP and B97-D/def2TZVP levels, respectively, and magnetic shieldings evaluated at the BHandH/6$311+\mathrm{G}(2 \mathrm{~d}, \mathrm{p})$ level (Tables S2-S5 in the ESI $\dagger$ ) by using Gaussian 09 Revision A.02 program. ${ }^{17}{ }^{1} \mathrm{H}$ magnetic shieldings were converted into relative shifts $\delta$ using the ${ }^{1} \mathrm{H}$ magnetic shielding in TMS computed at the same respective levels $(31.62 \mathrm{ppm}$ and 31.38 ppm for B3LYP and B97-D geometries, respectively). Selected calculations were also performed using a polarisable continuum model in the NMR calculation (in its 
integral equation formalism variant, IEF-PCM) ${ }^{18}$ using the parameters of benzene (dielectric constant 2.27). The QTAIM and NCI methods were carried out by using the AIMALL $^{19}$ and NCIPLOT $^{20}$ programs, respectively, on the B3LYP/def2-TZVP obtained electron densities.

The QM/MM protocol ${ }^{16}$ for optimising the crystal structure of $\mathbf{3}$ was carried out by using the Chemshell ${ }^{21}$ program linked to $\mathrm{ORCA}^{22}$ program and adapted as follows: A cluster with a radius of $42 \AA$ (containing 33,880 atoms/1210 molecules) was taken from the experimental crystal structure. Atomic charges from natural population analysis (NPA) for a free molecule $\mathbf{3}$ were obtained at a variety of DFT levels (different functional were used in the present work; see below) and were assigned to all other molecules of the built cluster. Surface charges were added on the boundaries of the cluster in order to emulate the remaining, infinite periodic system. The NPA charges were then refined self-consistently in a series of QM/MM single-point calculations for a single molecule 3 electrostatically embedded in the surrounding MM point charges (cluster and the cluster boundary charges were updated in each cycle self-consistently until the charges changed by less than $0.0001 \mathrm{au}$ ). Assigning van der Waals parameters from the UFF force field to all atoms in the cluster (to derive 6-12 Lennard-Jones potentials for all atom pairs), the NPA charges were refined again through a series of QM/MM geometry optimizations where only the central molecule was included in the QM region, while all other MM molecules were kept frozen. Finally the QM region was enlarged to encompass the central molecule and two neighboring molecules (affording a trimer as shown in Figure 6c) and QM/MM optimisations were carried out using the self-consistent charges determined previously. These calculations used BP86, B3LYP, revPBE ${ }^{23}$ and PW6B95 ${ }^{24}$ functionals with and without Grimme dispersion corrections (DFT-D3) $^{13,14}$ and with the def2-SVP and def2-TZVP basis sets. ${ }^{8}{ }^{1} \mathrm{H}$ NMR chemical shifts were calculated at the BHandH/6-311+G(2d,p) level for the B3LYP/def2TZVPoptimised trimer, embedded in the array of MM point charges (reported for the central molecule only). Relaxation of the QM region containing the trimer in the gas phase started from the QM/MM-optimised coordinates using ORCA.

\section{Acknowledgements}

The authors thank a Grant \# 2012/03933-5, São Paulo Research Foundation (FAPESP) for providing financial support for this research, for a scholarship [to RAC \#2011/01170-1, FAPESP). CNPq is also gratefully acknowledged for a fellowship (to RR) and a studentship (to RAC). We thank the Royal Society (Wolfson Merit Award for DO'H) and EaStCHEM (support for MB and access to a computing facility maintained by H. Früchtl). 


\section{References}

1 D. O’Hagan Chem. Soc. Rev. 2008, 37, 308.

2 R. Berger, G. Resnati, P. Metrangolo, E. Weberd, J. Hulliger Chem. Soc. Rev. 2011, 40, 3496.

3 A. J. Durie, A. M. Z. Alexandra, T. Lebl, P. Kirsch, D. O'Hagan Chem. Commun. 2011, 47, 8265 .

4 A. J. Durie, A. M. Z. Slwein, T. Lebl, P. Kirsch, D. O'Hagan Chem. Commun. 2012, $48,9643$.

5 A. J. Durie, T. Fujiwara, R. Cormanich, M. Bühl, A. M. Z. Slawin, D. O'Hagan, Chem. Eur. J., submitted (see Supporting Information for Review).

6 See e.g.: E. M. Engler, P. Laszlo J. Am. Chem. Soc. 1971, 93, 1317.

7 Benzene was used as model because it has very similar electronic properties as toluene, but lacks the conformational complexity in an adduct (i.e. relative positions of the additional Me group and the $\mathrm{F}$ atoms in the cyclohexane).

8 F. Weigend, R. Ahlrichs Phys. Chem. Chem. Phys. 2005, 7, 3297.

9 R. F. W. Bader, Atoms in Molecules: A Quantum Theory, Clarendon, Oxford, 1990.

10 E. Johnson, S. Keinan, P. Mori-Sánchez, J. Contreras-García, A. Cohen, W. Yang J. Am. Chem. Soc. 2010, 132, 6498.

11 T. V. Rybalova, I. Yu. Bagryanskaya J. Struct. Chem. 2009, 50, 741.

12 R. M. Osuna, V. Hernández, J. T. L. Navarrete, E. D’Oria, J. J. Novoa Theor. Chem. Acc. 2011, 128, 541.

13 S. Grimme, S. Ehrlich, L. Goerigk J. Comput. Chem. 2011, 32, 1456.

14 S. Grimme, J. Antony, S. Ehrlich, H. Krieg J. Chem. Phys. 2010, 132, 154104.

15 P. Lazzeretti, Prog. NMR Spectr. 2000, 36, 1.

16 R. Björnsson, M. Bühl J. Chem. Theory Comput. 2012, 8, 498.

17 Gaussian 09, Revision A.02, M. J. Frisch, G. W. Trucks, H. B. Schlegel, G. E. Scuseria, M. A. Robb, J. R. Cheeseman, G. Scalmani, V. Barone, B. Mennucci, G. A. Petersson, H. Nakatsuji, M. Caricato, X. Li, H. P. Hratchian, A. F. Izmaylov, J. Bloino, G. Zheng, J. L. Sonnenberg, M. Hada, M. Ehara, K. Toyota, R. Fukuda, J. Hasegawa, M. Ishida, T. Nakajima, Y. Honda, O. Kitao, H. Nakai, T. Vreven, J. A. Montgomery, Jr., J. E. Peralta, F. Ogliaro, M. Bearpark, J. J. Heyd, E. Brothers, K. N. Kudin, V. N. Staroverov, R. Kobayashi, J. Normand, K. Raghavachari, A. Rendell, J. C. Burant, S. S. Iyengar, J. Tomasi, M. Cossi, N. Rega, J. M. Millam, M. Klene, J. E. Knox, J. B. Cross, V. Bakken, C. Adamo, J. Jaramillo, R. Gomperts, R. 
E. Stratmann, O. Yazyev, A. J. Austin, R. Cammi, C. Pomelli, J. W. Ochterski, R. L. Martin, K. Morokuma, V. G. Zakrzewski, G. A. Voth, P. Salvador, J. J. Dannenberg,

S. Dapprich, A. D. Daniels, O. Farkas, J. B. Foresman, J. V. Ortiz, J. Cioslowski, and D. J. Fox, Gaussian, Inc., Wallingford CT, 2009.

18 G. Scalmani, M. J. Frisch, J. Chem. Phys., 2010, 132, 114110.

19 A. K. Todd, AIMAll (Version 13.05.06), 2013 (aim.tkgristmill.com).

20 J. Contreras-García, E. R. Johnson, S. Keinan, R. Chaudret, J.-P. Piquemal, D. N. Beratan, W. Yang J. Chem. Theor. Comput. 2011, 7,625.

21 ChemShell, a Computational Chemistry Shell, see www.chemshell.org.

22 F. Neese, ORCA; University of Bonn: Bonn, Germany, 2010. http://www.thch.unibonn.de/tc/orca.

23 Y. Zhang, W. Yang Phys. Rev. Lett.1998, 80, 890.

24 Y. Zhao, D. G. Truhlar J. Phys. Chem. A. 2005, 109, 5656. 
Table of Contents Graphic

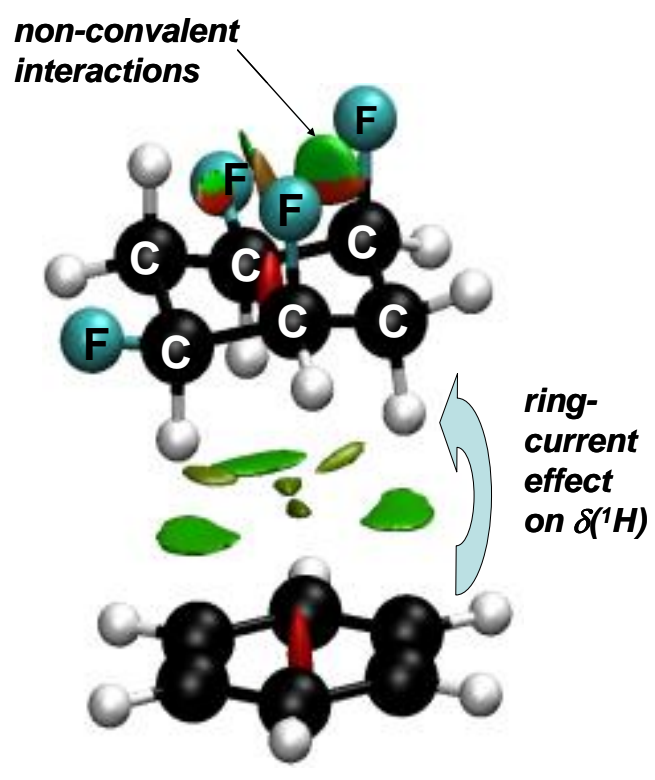

